\title{
Corrigendum: Proteome-wide profiling of protein assemblies by cross-linking mass spectrometry
}

\author{
Fan Liu, Dirk T S Rijkers, Harm Post \& Albert J R Heck \\ Nat. Methods 12, 1179-1184 (2015); published online 28 September 2015; corrected after print 17 February 2017
}

In the version of this article initially published, the number of interprotein cross-links was overstated, as protein grouping during cross-link categorization was not properly considered. 314 intraprotein cross-links were mistakenly annotated as interprotein cross-links if shared peptides between different protein entries were involved. The cross-links were reannotated after protein grouping, and cross-links were removed from contaminants (e.g., keratin).

The sentence in the abstract, "This approach allowed us to detect 2,179 unique cross-links (1,665 intraprotein cross-links at a 5\% false discovery rate (FDR) and 514 interprotein cross-links at 1\% FDR) in HeLa cell lysates," has been changed to "This approach allowed us to detect 2,426 unique cross-links at a 5\% FDR (2,013 intraprotein and 413 interprotein cross-links) or 1,822 cross-links at a 1\% FDR (1,622 intraprotein and 200 interprotein cross-links), indicating the detection of 326 or 134 protein-protein interactions at 5\% FDR or 1\% FDR, respectively, in HeLa cell lysates."

The sentence, "We unambiguously identified 2,179 cross-links (1,665 intraprotein cross-links at 5\% FDR and 514 interprotein cross-links at $1 \% \mathrm{FDR}$ ) in HeLa cell lysates using the whole human proteome database ( 40,000 entries) as the search space," has been changed to, "We unambiguously identified 2,426 total cross-links at 5\% FDR or 1,822 total cross-links at 1\% FDR in HeLa cell lysates using the whole human proteome database ( 40,000 entries) as the search space."

The sentence in the Results, "At 5\% FDR, 2,473 unique cross-links were identified via the combined CID-ETD strategy, whereas 1,113 unique cross-links were identified in CID-only analysis, which corresponds to a difference of more than twofold (Fig. 2a)," has been changed to, "At $5 \%$ FDR, 2,426 unique cross-links were identified via the combined CID-ETD strategy, whereas 1,089 unique cross-links were identified in CID-only analysis, which corresponds to a difference of more than twofold (Fig. 2a)."

The sentence in the Results, "When we repeated the analysis with a more stringent FDR cutoff of 1\%, the discrepancy between CID-ETD and CID-only data increased to threefold (1,867 cross-links identified with CID-ETD and 594 cross-links identified with CID only) (Fig. 2a)," has been changed to, "When we repeated the analysis with a more stringent FDR cutoff of 1\%, the discrepancy between CID-ETD and CID-only data increased to threefold (1,822 cross-links identified with CID-ETD and 580 cross-links identified with CID only) (Fig. 2a)"”

The sentence in the Results, "On the basis of the statistical analysis of the distribution of target and decoy hits, we decided to use a more stringent FDR cutoff for interprotein cross-links than for intraprotein cross-links. Thus the reported 2,179 cross-links were filtered at $5 \%$ FDR for intraprotein cross-links (1,665 cross-links) and at 1\% FDR for interprotein cross-links (514 cross-links) (Fig. 2a)," has been changed to, "On the basis of the statistical analysis of the distribution of target and decoy hits, we suggest using a more stringent FDR cutoff for interprotein cross-links compared than for intraprotein cross-links. All protein-protein interactions we detected based on our interprotein cross-link findings are reported in Supplementary Data 2."

We added this sentence about our reanalysis in the Online Methods section: "Cross-links were categorized as 'interprotein' if the two linked peptides derived from two different protein groups (i.e., a set of proteins containing shared peptides); otherwise, the cross-link was annotated as 'intraprotein'. We note that if homo-oligomers or complexes wherein multiple copies of a single protein are present (co-)exist, cross-links comprising two peptides of the same protein group may either be 'intermolecular' (between homo-oligomeric subunits) or 'intramolecular' (within one subunit)." We also added the sentences, "Cross-links from contaminants (e.g., keratin) were removed" and "Protein-protein interactions detected in our cross-linking data set are reported in Supplementary Data 2. Annotated spectra for interprotein cross-links are available in Supplementary Data 3; annotated spectra for intraprotein cross-links are available in Supplementary Data 4."

Figure 2 has been replaced to correctly reflect the results of our updated reanalysis described above.

The Supplementary Data "all 2,179 crosslinks" table has been replaced with a new table, named Supplementary Data 1, which reports the crosslinks identified in our reanalysis. Another table, named Supplementary Data 2, has been added to report the protein-protein interactions detected in the cross-linking data set. The two folders containing annotated mass spectra in the "Supplementary Data" zip file have been renamed Supplementary Data 3 and 4.

These errors have been corrected in the PDF and HTML versions of the article. 